\title{
EXPERIENCIA EN LA CORRECCIÓN QUIRÚRGICA DE LA INCONTINENCIA DE ORINA DE ESFUERZO CON TÉCNICA TOT (TRANS-OBTURATOR-TAPE).
}

\author{
Jack Pardo Schanz $z^{(1,2)}$, Paolo Ricci Arriola(1,2), Vicente Solà Dalenz² y Ximena Tacla Fernández
}

'Departamento de Ginecología y Obstetricia, Hospital Barros Luco-Trudeau. Santiago de Chile.

2Unidad de Uroginecología y Cirugía Vaginal, Departamento de Ginecología y Obstetricia Clínica Las Condes.

Santiago de Chile.

\begin{abstract}
Resumen.- OBJETIVO : Evaluar la seguridad y eficacia de la técnica quirúrgica de TOT en el tratamiento de la incontinencia de orina de esfuerzo femenina.

MÉTODO: Seguimiento prospectivo de 49 pacientes sometidas a TOT, en la Unidad de Ginecología del Departamento de Ginecología y Obstetricia del Hospital Barros Luco-Trudeau, entre noviembre 2003 y enero 2005. Se utilizó malla de polipropileno, macroporo, monofilamento (Prolene). Mediana de edad 52 años, paridad 3. Se completó un período de observación hasta noviembre de 2005.

RESULTADO: Mediana de tiempo operatorio de TOT, 15 minutos. En 32 (65\%) se asoció otra cirugía ginecológica. En el intraoperatorio se presentó una lesión de vejiga. En el postoperatorio inmediato un caso de infección del tracto urinario bajo, y otro de pielonefritis aguda. Al finalizar el período de observación, se obtiene cura en $47(96 \%)$ casos, mejoría $1(2 \%)$ y falla $1(2 \%)$. El caso de falla resulta después de un traumatismo a los tres meses.
\end{abstract}

Paolo Ricci Arriola

Hospital Barros Luco Trudeau,

Avenida José Miguel Carrera 3204, San Miguel, Santiago de Chile. (Chile) pricci@vtr.net

Trabajo recibido: 16 de noviembre 2005
CONCLUSIONES: EI TOT se presenta como una técnica eficaz en la corrección de la IOE. Además es de mayor facilidad que el TVT. Aunque escasas, no está exento de complicaciones.

Palabras clave: Incontinencia de orina de esfuerzo (IOE). Cinta libre de tensión transobturador (TOT).

Summary.- OBJECTIVES: To evaluate the safety and efficacy of the TOT surgical technique in the treatment of female stress urinary incontinence (SUI).

METHODS: Prospective study of 49 patients undergoing TOT at the Gynecology Unit of the Obstetrics and Gynecology Department in Barros Luco-Trudeau Hospital between November 2003 and January 2005. The mesh used was polypropylene, macropore, monofilament (Prolene). Median age was 52 years. The observational phase was completed in November 2005.

RESULTS: Median operative time was 15 minutes. Thirtytwo cases (65\%) underwent a second gynecologic surgery. One patient had an intraoperative bladder lesion. In the immediate postoperative period there was one case of lower urinary tract infection and another acute pyelonephritis. At the end of the observation phase 47 (96\%) patients were cured, 1 (2\%) improved, and 1 (2\%) had a failure. The failure appeared after a trauma three months after surgery.

CONCLUSIONS: The TOT is an effective technique for the correction of SUI. It is easier than TVT. Although rare, it is not free of complications.

Keywords: Stress urinary incontinence (SUI). Tension-free transobturator tape (TOT). 


\section{INTRODUCCIÓN}

En 1996 Ulmstein (1) publica la técnica de TVT para la corrección de la incontinencia de orina de esfuerzo, una cirugía mínimamente invasiva, pero con la desventaja de requerir cistoscopia intraoperatoria. Además no exenta de riesgos como lesión vesical, de vías urinarias e intestinal (2-6). Durante el 2001 buscando una mayor simplificación, Delorme (7) describe la técnica de TOT (trans-obturator-tape). La principal ventaja de esta técnica es la posición de la cinta, que es más anatómica que el TVT. La cinta duplica la posición de los ligamentos pubouretrales. Las agujas no pasan por el espacio retropúbico, posee menos sangrado, ausencia de incisiones abdominales, disminución del riesgo de injuria vesical $(8,9)$ e intestinal, y no requiere cistoscopia. Constituyéndose como una cirugía de mayor facilidad y rapidez en su ejecución, respecto a TVT. (10) Al comparar la eficiencia en la corrección de la incontinencia de orina de esfuerzo, el TOT parece ser igualmente efectivo que TVT. (1 11 )

Con el objetivo de evaluar la seguridad y eficacia del TOT en el tratamiento de la incontinencia de orina de esfuerzo en la mujer, analizamos nuestra experiencia en 49 casos.

\section{MÉTODOS}

Protocolo de Estudio: Seguimiento prospectivo de 49 pacientes, ingresadas al Departamento de Ginecología del Hospital Barros Luco-Trudeau, para corrección de la IOE por medio de TOT, entre noviembre del 2003 y enero del 2005.

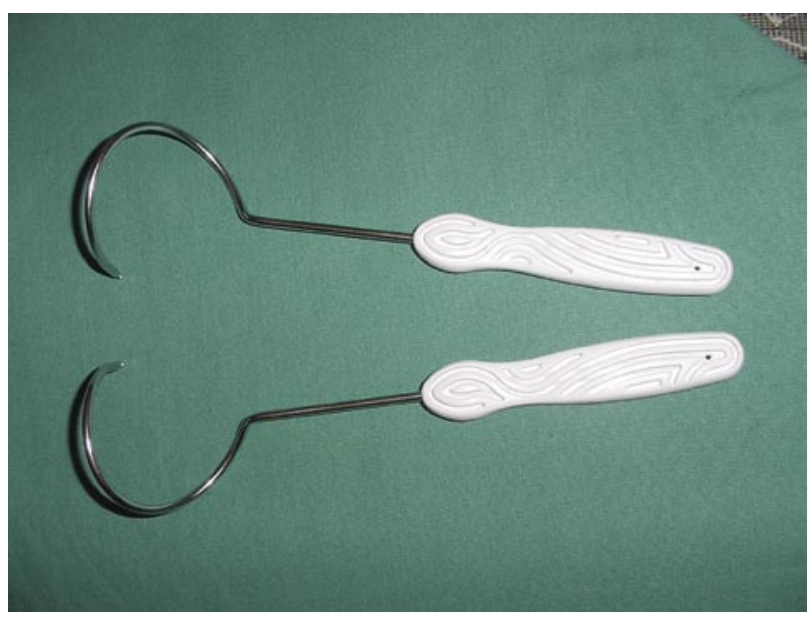

FIGURA 1. Agujas utilizadas en corrección de incontinencia de orina de esfuerzo, con técnica quirúrgica TOT.
Criterio de inclusión: Todas las pacientes ingresadas al estudio debían presentar incontinencia de orina de esfuerzo al menos por un año y de carácter permanente.

Criterio de curación, mejoría y fracaso: Se clasificó el resultado de la cirugía, según el número de episodios de incontinencia de orina registrados durante el período de observación. Se considera cura a la ausencia de incontinencia. Mejoría a la presencia de episodios de incontinencia menos que una vez cada dos semanas. Falla a la presencia de episodios de incontinencia más de una vez por semana.(12)

Período de observación o seguimiento: hasta noviembre de 2005.

Técnica quirúrgica específica: Se utilizó la técnica de TOT descrita originalmente por Delorme (7). Se usó cinta sintética de polipropileno monofilamento macroporo (Prolene). Cada cinta medía $10 \mathrm{~mm}$

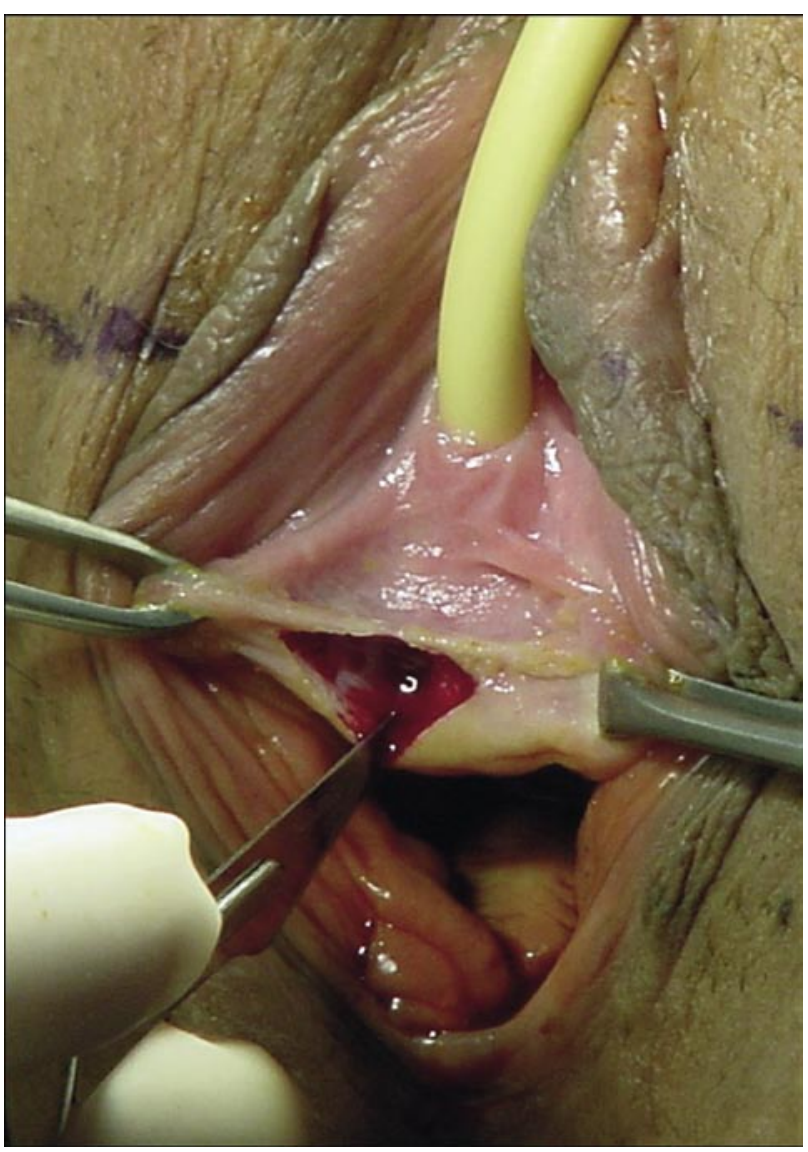

FIGURA 2. Se abre la mucosa de la pared vaginal anterior a un centímetro bajo el meato urinario. La incisión es de un centímetro. 
de ancho y $30 \mathrm{cms}$ de largo. Para su introducción se usaron dos agujas helicoidales, una para cada lado. Figura 1.

Se utilizó anestesia raquídea para realizar el procedimiento quirúrgico, y se situó a la paciente en la camilla operatoria en posición ginecológica.

Se realizó aseptización vaginal y perivaginal con povidona yodada. Se colocó una sonda Foley para vaciar la vejiga, la que se dejó hasta el postoperatorio.

Paso 1: Se marcan los puntos de salida de las agujas. Para ello se traza línea desde el clítori hasta interceptar el pliegue inguinal.

Paso 2: Se abre la mucosa de la pared vaginal anterior a un centímetro bajo el meato urinario. La incisión es de un centímetro. Se diseca lateralmente y a ambos lados de la zona media uretral con tijera

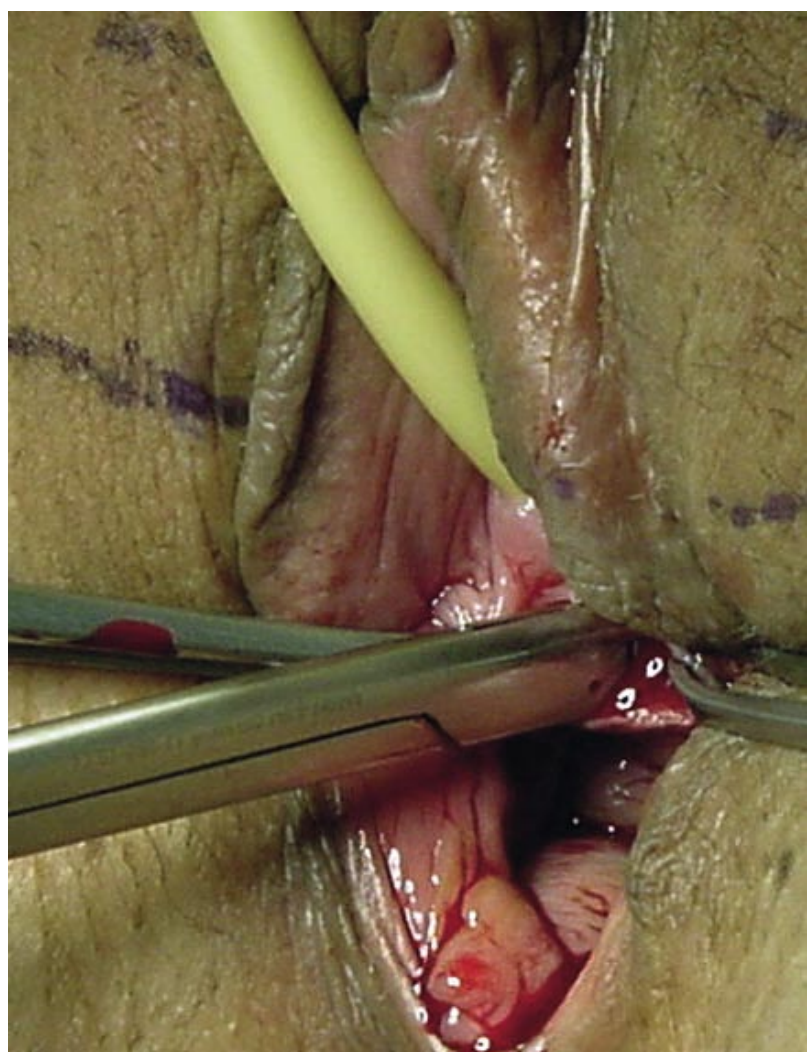

FIGURA 3. Se diseca lateralmente y a ambos lados de la zona media uretral con tijera fina, con un ángulo de 45 grados a la línea media, orientándose inmediatamente por debajo del plano horizontal de la mucosa, y en dirección a los puntos de salida descritos en el paso 1 fina, con un ángulo de 45 grados a la línea media, orientándose inmediatamente por debajo del plano horizontal de la mucosa, y en dirección a los puntos de salida descritos en el paso 1. (Figuras 2 y 3 ).

Paso 3: Se realiza incisión de $5 \mathrm{~mm}$ en piel de zona inguinal previamente marcada.

Paso 4: Se introduce la aguja helicoidal, dirigiéndola con dedo localizado en la incisión vaginal. La aguja traspasa la membrana obturadora. Se extrae el extremo de la aguja a través de la incisión vaginal. Ver (Figuras 4 y 5 ).

Paso 5: Se une la cinta a la aguja y se extrae por la incisión inguinal, realizando el paso inverso a lo descrito en el punto anterior. Se repite el punto 2 al 5 en el lado contraleteral.

Paso 6: Se ajusta la tensión de la malla. Para lo cual se introduce una tijera entre la uretra y la malla. Luego se corta la cinta a nivel subcutáneo, y se sutura con un solo punto la piel. Finalmente se sutura la mucosa vaginal. (Figura 6).

En los casos en que asoció otra cirugía ginecológica, se realizó en un tiempo posterior al TOT.

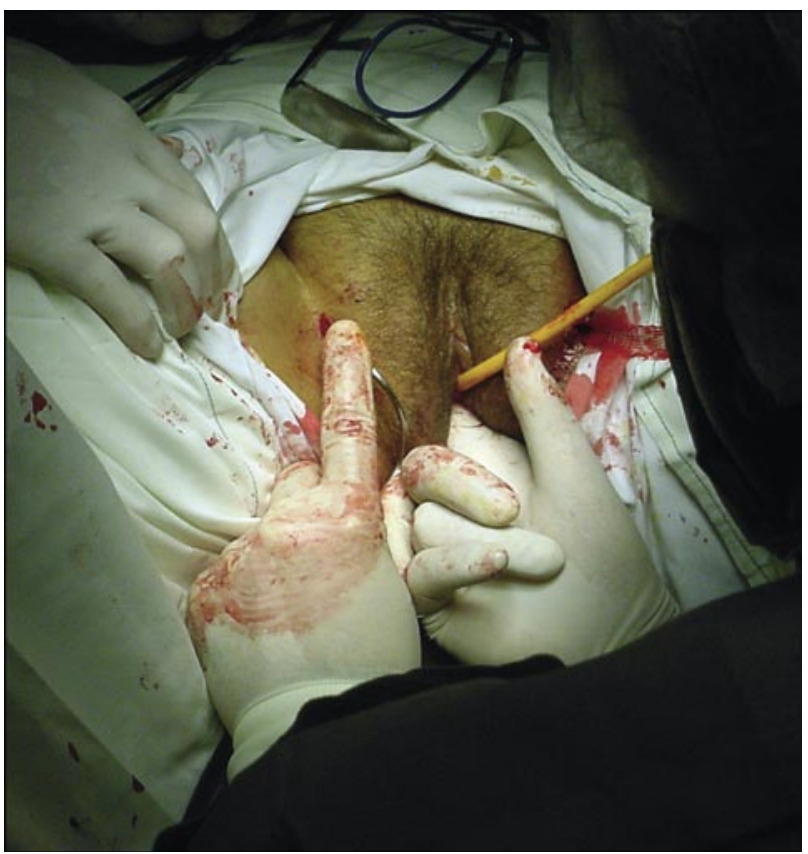

FIGURA 4. Se introduce la aguja helicoidal, dirigiéndola con dedo localizado en la incisión vaginal. La aguja traspasa la membrana obturadora. Se extrae el extremo de la aguja a través de la incisión vaginal. 
A todas las pacientes se les explicó previamente, en qué consistía el TOT y en los casos correspondientes, el procedimiento quirúrgico concomitante a realizar. Todas firmaron un consentimiento informado.

Se utilizó cefazolina 1 gramo endovenoso intraoperatorio como profilaxis. La analgesia postoperatoria fue intramuscular el primer día y vía oral a partir del segundo día, con refuerzo intramuscular en caso necesario.

En los casos de TOT sin otra cirugía asociada, se retiró la sonda Foley inmediatamente en el postoperatorio. En los que se asoció histerectomía vaginal y/o colpoperineoplastía anterior y/o posterior, se retiró 12 horas después.

Se dio alta hospitalaria al tercer o cuarto día dependiendo de la cirugía asociada.

Se controló en consulta ambulatoria entre los 7 y 14 días, al mes, a los 3 y 6 meses. Luego en forma telefónica, indicando control ambulatorio en caso de complicación o alteración de la continencia alcanzada.

Características del grupo en estudio: La mediana de edad fue de 52 años, con rango entre 34 y

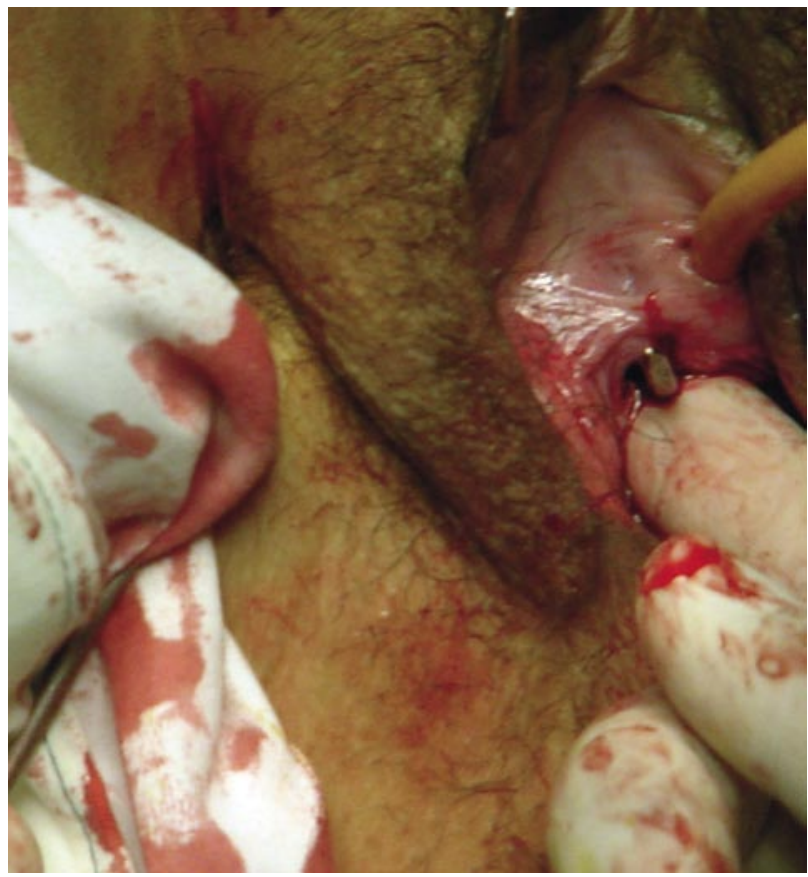

FIGURA 5

Se extrae el extremo de la aguja a través de la incisión vaginal.
78 años. Paridad mediana 3, rango 1 a 8 . Mediana de presentación de la incontinencia de orina de esfuerzo, 24 meses. (Tabla I).

\section{RESULTADOS}

De los 49 pacientes a los que se realizó TOT, en $32(65 \%)$ se asoció otra cirugía ginecológica. Las cirugías asociadas fueron: colpoperineoplastía anterior, colpoperineoplastía posterior, histerectomía vaginal, traquelectomía y biopsia vulvar.

En 20 casos se asociaron 2 cirugías, en 7 una, y en 5 tres cirugías. La asociación mayor fue TOT a colpoperineoplastía anterior y posterior. (Tabla II).

El tiempo operatorio del TOT fue de 15 minutos mediana, con rango entre 10 y 24 minutos.

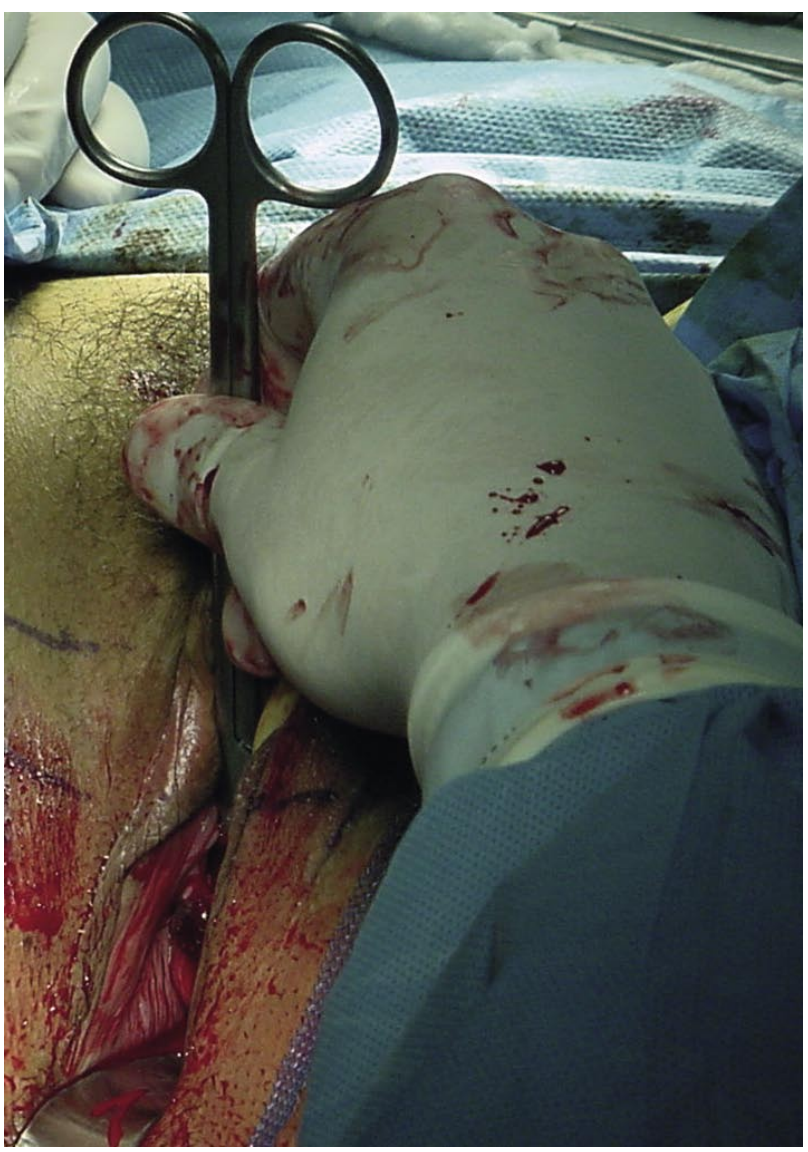

FIGURA 6. Se ajusta la tensión de la malla. Para lo cual se introduce una tijera entre la uretra y la malla. Luego se corta la cinta a nivel subcutáneo, y se sutura con un solo punto la piel. Finalmente se sutura la mucosa vaginal. 


\begin{tabular}{|ll|}
\hline Total pacientes en estudio: & 49 pacientes \\
Criterio de Inclusión: & IOE presentada por un año a lo menos y de \\
& carácter permanente. \\
& TOT para corrección de IOE. \\
Intervención quirúrgica: & Noviembre del 2003 y enero del 2005. \\
Período de intervenciones: & Hsta noviembre de 2005. \\
Edad de pacientes: & Mediana 52 años (rango entre 34 y 78 años) \\
Tiempo de presentación de IOE: & Mediana 24 meses. \\
\hline
\end{tabular}

IOE: Incontinencia de orina de esfuerzo.

TOT: trans-obturator-tape

Complicaciones intraoperatorias: Se presentó un caso, que correspondió a una lesión de vejiga con la tijera al disecar el espacio vesico-vaginal hacia el agujero obturador. Fue de 2 centímetros y se suturó en un plano con vycril 3-0 por vía vaginal. Se dejó Sonda Foley por 10 días con recuperación completa, y sin presentar nueva complicación posterior. (Tabla III).

Complicaciones del postoperatorio inmediato (hasta 7 días después de la cirugía): Se presentaron dos casos de infección urinaria. Uno correspondió a una infección de tracto urinario bajo, que se trató con antibióticos vía oral. El otro caso presentó una pielonefritis aguda, que se trató con antibióticos endovenosos con buena respuesta. Sin relación al TOT, se presentó un caso de hematoma de la cúpula vaginal, con drenaje espontáneo, en una paciente en la que se asoció histerectomía vaginal. (Tabla III).

Complicaciones del postoperatorio tardío (después de 7 días de la cirugía): En una paciente con cura de su IOE, al tercer mes de operada sufre una violenta caída. Reiniciando inmediatamente después de este evento la sintomatología de incontinencia de esfuerzo, considerándose como falla. Al examinarla se sospechó desplazamiento de la cinta. (Tabla III).

Solución de la incontinencia de orina: De las 49 pacientes, al mes se constató curación de la incontinencia de esfuerzo en $48(98 \%)$ de ellas. El otro caso presentó mejoría (1 caso, $2 \%$ ), quedando con incontinencia sólo a grandes esfuerzos.

Durante los controles ambulatorios después del primer mes (3 y 6 meses) no hubo diferencia en la condición de su continencia alcanzada con el TOT inicialmente. Excepto por el caso que después de un trauma pierde su condición de cura, para recuperar su incontinencia previa (falla).

Al completar el período de seguimiento a noviembre de 2005, 47 pacientes cumplían criterio de curación, 1 de mejoría, y 1 con falla (después de traumatismo a los 3 meses). (Tabla IV).

\section{TABLA II. CIRUGÍAS ASOCIADAS A TOT.}

De los 49 pacientes con TOT, en $32(65 \%)$ se asoció otra cirugía ginecológica.

Colpoperineoplastía anterior:

Colpoperineoplastía posterior:

Colpoperineoplastía anterior y posterior:

Histerectomía vaginal + Colpoperineoplastía posterior:

Histerectomía vaginal + Colpoperineoplastía anterior y posterior:

Traquelectomía:

Biopsia Vulvar:
2

2

15

5

5

1

2

En 20 casos se asociaron 2 cirugías, en 7 una, y en 5 tres cirugías. 
TABLA III. COMPLICACIONES Y RESOLUCIÓN.

\section{Complicaciones Intraoperatorias}

\section{Total: 1 caso}

Tipo: Lesión de vejiga con la tijera al disecar el espacio vesico-vaginal hacia el agujero obturatriz.

Resolución: sutura en un plano con vycril 3-0 por vía vaginal. Sonda Foley por

10 días con recuperación completa, y sin presentar nueva complicación posterior.

\section{Complicaciones del Postoperatorio Inmediato}

Total: 2 casos

Tipo: Infección urinaria. Una infección de tracto urinario bajo y una pielonefritis.

Resolución: Antibioticoterapia.

\section{Complicaciones del Postoperatorio tardío}

\section{Total: 1 caso}

Tipo: Pérdida de la continencia lograda después del TOT, producto de una caída al tercer mes. Al examinarla se sospechó desplazamiento de la cinta.

Resolución: Se programa nueva cirugía.

- Complicaciones del postoperatorio inmediato consideradas hasta los siete días después de la intervención.

- Complicaciones del Postoperatorio tardío consideradas después de los siete días desde la intervención.

\section{CONCLUSIONES}

Indudablemente se trata de un procedimiento quirúrgico de "sling" que simplifica la técnica quirúrgica al compararlo con TVT. Sin embargo no está totalmente exento de riesgos, como es la lesión de la vejiga por el paso de la aguja, aunque teóricamente sería menor la probabilidad respecto a su antecesor TVT. La primera publicación que presentaba esta complicación fue realizada por Hermiu (9) el 2003. En la lesión vesical presentada en nuestra casuística, la causa fue la disección con tijera de la fascia vesico-

Total: 49 pacientes. Seguimiento hasta noviembre 2005.

Seguimiento máximo: 24 meses

Promedio de seguimiento: 17 meses

Seguimiento mínimo: 10 meses

$\begin{array}{lll} & \text { Total } & \text { Porcentaje (\%) } \\ \text { Cura* } & 47 & 96 \\ \text { Mejoría** } & 1 & 2 \\ \text { Falla*** } & 1 & 2\end{array}$

*Cura: ausencia de incontinencia

** Mejoría: episodios de incontinencia < que 1 vez cada 2 semanas

***Falla: episodios de incontinencia > que 1 vez por semana

Caso de falla corresponde a paciente con cura de IOE que al tercer mes de postoperatorio, como resultado de traumatismo pierde su continencia. 
vaginal hacia el agujero obturador. Al analizar esta complicación debemos mencionar que se debió a un insuficiente vaciado vesical con sonda Foley, sumado a un severo cistocele. En base a esta experiencia, debemos insistir en el vaciamiento completo de la vejiga antes de iniciar la disección con tijera, a fin de evitar esta complicación, sobretodo en pacientes con cistocele moderado o severo.

Resulta interesante analizar el alto porcentaje de cura de la incontinencia de esfuerzo alcanzada con esta técnica.

Igualmente es interesante destacar y analizar el caso con respuesta total (cura), que frente a una caída reinicia los síntomas de incontinencia de orina de esfuerzo. No encontrándose caso similar en otras publicaciones, que deducimos por el examen vaginal practicado, se trataría de una alteración en la ubicación de la cinta de polipropileno, favorecido por una sobreabducción forzada de la extremidad inferior frente a una violenta caída, dejando de cumplir su función.

En nuestra experiencia utilizamos malla de polipropileno, macroporo, monofilamento de Prolene, para facilitar el acceso a esta técnica con costos menores para las pacientes del sector público de salud, frente a otras como Gynemesh o Atrium que son de costo superior. Aún cuando en los tres casos mencionados, se trata de mallas clasificadas quirúrgicamente como Tipo 1 (13), es decir mallas de polipropileno, monofilamento y macroporo, presentan diferencias en cuanto a presión de ruptura (psi), espesor, flexibilidad $(\mathrm{mg} / \mathrm{cm})$ y fuerza tensil (lb). Lo que teóricamente podría llevar a resultados diferentes según la malla utilizada.

Finalmente podemos afirmar que el TOT se presenta como una técnica segura si se toman medidas como un completo vaciamiento de la vejiga. Ya que la perforación vesical aún cuando es menos probable de realizar por el paso de la aguja, al compararla con el TVT (razón por la cual no es necesario realizar una cistoscopia intraoperatoria en TOT), constituye una de las potenciales complicaciones, como ocurrió en una paciente de nuestra casuística. Aún cuando se trató de una perforación realizada con la tijera de disección y de fácil resolución.

Además es eficaz en la corrección de la $\mathrm{IOE}$, como ha sido demostrado en otros trabajos $(7,8,10,11)$. Y que en nuestra experiencia permitió alcanzar altos porcentajes de cura y mejoría, los que se mantuvieron a lo largo del seguimiento (a excepción del caso de falla después de traumatismo). Por otro lado no podemos dejar de mencionar que se trata de una técnica fácil de aprender, con una corta curva de aprendizaje. Sin embargo, la alta asociación de TOT a otras cirugías del suelo pélvico tales como histerectomía vaginal y corrección de prolapso, hace necesario en nuestra opinión, que esta técnica quirúrgica sea realizada por ginecólogos con experiencia en cirugía vaginal.

Además se trata de una técnica quirúrgica que posee una alta aceptación por los pacientes debido a que es una cirugía mínimamente invasiva.

\section{BIBLIOGRAFIA y LECTURAS RECOMENDADAS ( ${ }^{*}$ lectura de interés $y^{* *}$ lectura fundamental)}

1. ULMSTEN, U.; HENRIKSSON, L.; JOHNSON, P. y cols.: "An ambulatory surgical procedure under local anesthesia for treatment of female urinary incontinence". Int. Urogynecol. J. Pelvic Floor Dysfunct., 7: 81, 1996.

2. ULMSTEN, U.; FALCONER, C.; JOHNSON, P. y cols.: "A multicenter study of tension-free vaginal tape (TVT) for surgical treatment of stress urinary incontinence". Int. Urogynecol. J. Pelvic Floor Dysfunct., 9: 210, 1998.

3. ALLAHDIN, S.; McKINLEY, C.; MAHMOOD, T.A. y cols.: "Tension-free vaginal tape: 162 cases in a district general hospital". J. Obstet. Gynaecol., 24: 539, 2004.

4. REZAPOUR, M.; ULMSTEN, U.: "Tension-free vaginal tape (TVT) in women with mixed urinary incontinence-a long-term follow-up". Int. J. Urogynecol. J. Pelvic Floor Dysfunct., 12: 15, 2001.

5. ABOUASSALY, R.; STEINBERG, J.R.; LEMIEUX, M. y cols.: "Complications of tensionfree vaginal tape surgery: a multi-institutional review". BJU Int., 94: 110, 2004.

6. JIMENEZ, C.J.; HUALDE, A.A.; GONZALEZ DE GARIBAY, A.S. y cols.: "TVT: three years of experience". Actas Urol. Esp., 28: 13, 2004.

7. DELORME, E.: "Transobturator urethral suspension: mini-invasive procedure in the treatment of stress urinary incontinence in women". Prog. Urol., 11: 1306, 2001.

8. DARGENT, D.; BRETONE, S.; GEORGE, P.; MELLIERE, G.: "Insertion of a suburethral sling through the obturating membrane for treatment of female urinary incontinence". Gynecol. Obstet. Fertil., 30: 57, 2002.

9. HERMIEU, J.F.; MESSAS, A.; DELMAS, V. y cols.: "Bladder injury after trans-obturator tape". Prog. Urol., 13: 115, 2003. 
10. MELLIER, G.; BENAYED, B.; BRETONES, S. y cols.: "Suburethral tape via the obturator route: is the TOT a simplification of the TVT?". Int. Urogynecol. J. Pelvic Floor Dysfunct., 15: 227, 2004.

11. DE TAYRAC, R.; DEFFIEUX, X.; DROUPY, S. y cols.: "A prospective randomized trial comparing tension-free vaginal tape and transobturator suburethral tape for surgical treatment of stress urinary incontinence". Am. J. Obstet. Gynecol., 190: 602, 2004.

12. BLAIVAS, J.G.; JACOBS, B.Z.: "Pubovaginal fascial sling for the treatment of complicated stress urinary incontinence". J. Urol., 145: 1214, 1991.

13. BIRCH, C.; FYNES, M.M.: "The role of synthetic and biological prothesis in reconstructive pelvic floor surgery". Curr. Opin. Gynecol., 14: 527, 2002. 